\title{
A Pixelated Positron Timing Counter with Fast Plastic Scintillator Readout by SiPMs for the MEG II
}

\section{Miki Nishimura*†}

The University of Tokyo

E-mail: nmiki@icepp.s.u-tokyo.ac.jp

The MEG experiment has searched for the $\mu^{+} \rightarrow e^{+}+\gamma$ decay setting a limit on the branching ratio down to $5.7 \times 10^{-13}$. This decay is basically forbidden in the Standard Model and any detection would be evidence of new physics. A MEG upgrade (MEG II) is planned to push the limit down to $5 \times 10^{-14}$. For this upgrade, substantial improvements in time, energy, and position resolutions are required. The positron emission time will be measured by a Timing Counter of novel design. It is segmented into 512 small plastic scintillator counters readout by SiPMs. The positron will hit multiple counters, each of them providing a measurement of time. The combination of these information will result in a improved resolution. This concept was tested with prototype counters, with single counter resolution of 75-80 ps, with the positron beam at LNF (Laboratori Nzaionali di Frascati, INFN), obtaining a combined resolution $\lesssim 40$ ps with six counters. Moreover, the single counter design has been optimized bringing the single counter resolution down to 50-60 ps, leading to the expectation of an improved combined resolution.

Technology and Instrumentation in Particle Physics 2014,

2-6 June, 2014

Amsterdam, the Netherlands

*Speaker.

${ }^{\dagger}$ on behalf of the MEG II collaboration 


\section{Introduction}

Charged lepton flavor violation is very sensitive to new physics Beyond Standard Model (BSM). Many extensions predict large branching ratio of $\mu^{+} \rightarrow e^{+}+\gamma$, well beyond the SM expectations. The upper limit to this decay was set by the MEG experiment to $5.7 \times 10^{-13}$ [1]. MEG is located at Paul Scherrer Institute (PSI) in Switzerland to use the world most intense DC $\mu^{+}$beam. The $\mu^{+} \rightarrow e^{+}+\gamma$ decay has the simple kinematics of a two body decay: monochromatic positrons and photons emitted back to back. In order to extract these decays from background, excellent time, energy, and position resolution are required. MEG is based on a LXe photon detector and a positron spectrometer embedded in a gradient magnetic field. The spectrometer consists of a set of drift chambers and a timing counter employing scintillator bars readout by PMTs.

However, with the resolution of MEG, the improvement in sensitivity is limited by the background. Furthermore the existing spectrometer cannot cope with an increased muon decay rate. To overcome these limitations, an upgrade of the detector is planned.

\section{New Positron Timing Counter}

A positron timing counter based on a novel concept is planned to obtain an improved time resolution and to cope with the planned increased positron rate. It is segmented into many small plastic scintillator counters readout by SiPMs on each side. Fig. 1 and Fig. 2 show an overview of the timing counter and the design of single counter. Individual counters consist of 90-120 $\times 40 \times 5 \mathrm{~mm}^{3}$ fast plastic scintillator (BC422, BC418, or EJ232) readout on each side by 3-6 AdvanSiD near-ultravoilet sensitive SiPMs (ASD-NUV3S-P50) connected in series. The counter signal directly passes through long cable. After long cable it is processed by a voltage amplifier and a shaper performing pole-zero cancellation and subsequently sampled and digitized by the waveform digitizer Domino Ring Sampler (DRS4) [2].

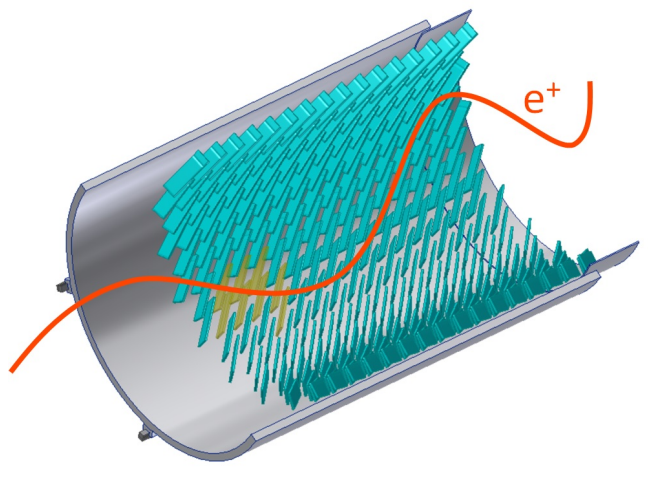

Figure 1: New pixelated positron timing counter overview. Orange line shows a simulated signal positron trajectory. Positron penetrates several counters.

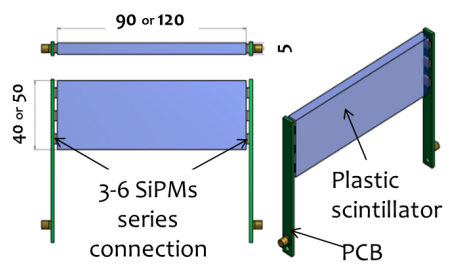

Figure 2: Counter design. Counter consists of plastic scintillator and SiPMs connected in series.

This design has several advantages: SiPMs can operate in high magnetic field, the pile up in a high rate environment is reduced due to the small size of the counter, several counters are crossed by a positron allowing multiple measurement of the positron time, that can be combined to improve the resolution down to $\sim 30 \mathrm{ps}$. 


\section{Prototype Test}

\section{Beam Test}

To prove the concept that the overall time resolution can be improved with multi-hit measurements, we tested a multi-counter prototype detector at Beam Test Facility in LNF, Frascati with a $48 \mathrm{MeV}$ positron beam, an energy close to the signal positron in the experiment. The bunch width is $10 \mathrm{~ns}$ and the number of positrons per bunch is $1-3$. Because the bunch width is short compared with the pile up rate in MEG II $(\sim 100 \mathrm{kHz})$, we selected only 1 positron events by analysis cut. The positron beam first hits a reference counter, used for time reference and trigger, consisting of a $5 \times 5 \times 5 \mathrm{~mm}^{3}$ scintillator (BC422) and readout by a Hamamatsu SiPM (S10362-33-050C). It is followed by the prototype counters. We prepared 8 counters with Hamamatsu SiPMs (S10943$2547(\mathrm{X}))$ and 6 with AdvanSiD SiPMs. Detailed study of these SiPM properties and comparison were carried out in [3]. Each counter consists of $90 \times 40 \times 5 \mathrm{~mm}^{3}$ BC418 scintillator readout by 3 SiPMs on each side. The counter signals are transmitted through 32-ns cable to voltage amplifiers and then digitized by 6 DRSs. Each channel jitter is adjusted with $\sim 25$ ps precision using sine wave clock.

The performances of the single counters were checked first, obtaining $\sim 75$ and $\sim 80$ ps for Hamamatsu and AdvanSiD, respectively, as shown in Fig.3. These results are consistent with those obtained in the laboratory with a ${ }^{90} \mathrm{Sr}$ source.

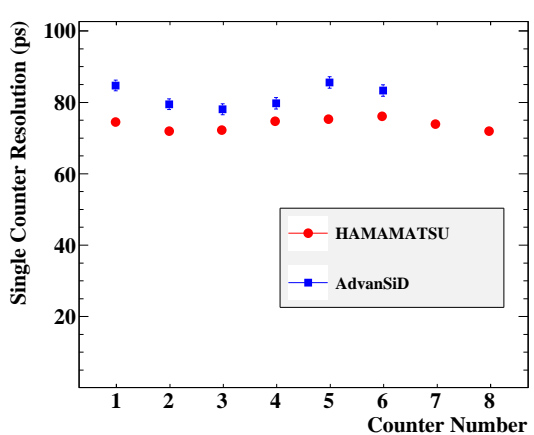

Figure 3: Single counter resolution. Every Hamamatsu and AdvanSiD counter perform with similar resolution.

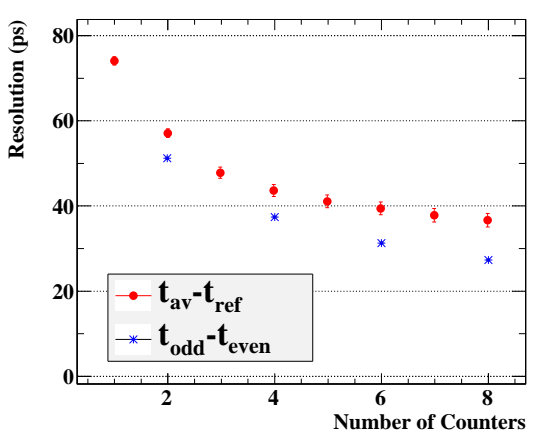

Figure 4: Overall resolution as a function of the number of counters (preliminary). Overall resolution improves with the number of hit counters.

The overall time resolution is evaluated in following two different approaches; both results (preliminary) are shown in Fig.4 In the first approach, the average time of hit counters is subtracted from the reference time. This analysis is similar to the real MEG II time reconstruction, $T_{e}-T_{\gamma}$. In the second approach, the even-odd analysis, the average time of even counters is subtracted from the average time of odd counters. It indicates the intrinsic resolution free from the reference counter. With six hit counters, comparable with the expected average counters in MEG II, the first approach resulted in a resolution $\sim 39$ ps. The second approach resulted in $\sim 30$ ps. The difference between the intrinsic resolution and the practical resolution is observed. The reason is under investigation. However, excellent overall time resolution $\lesssim 40$ ps was obtained. The result shows the effectiveness of the multiple hit approach. 


\section{Single Counter Design Optimization}

In the beam test, we proved the multiple hit approach and obtained excellent time resolution. Further development of single counter design, in particular optimization of the scintillator type and the number of SiPMs, are expected to improve this performance.

The choice of scintillator is a trade-off between rise time and light yield. We tested two type of scintillator: BC418 and BC422. Attenuation length is $100 \mathrm{~cm}$ and $8 \mathrm{~cm}$ and rise time is $0.5 \mathrm{~ns}$ and $0.35 \mathrm{~ns}$, respectively. Though $\mathrm{BC} 418$ was used in the beam test, the result shown in Fig. 5 indicates that $\mathrm{BC} 422$ has better time resolution.

The number of SiPMs in the beam test is three on each side. However we can increase the sensor coverage by using more SiPMs, increasing the photon collection efficiency. Therefore, we can expect to improve the single counter time resolution, which depends on the photo-electron statistic. We tested BC422 single counters with three, five, or six SiPMs on each side. As shown in Fig. 6, counters with more SiPMs have better resolution.

Finally, the single counter resolution improves from $75-80$ ps to $50-60$ ps with BC422 and 6 SiPMs on each side. We plan to test this optimized counter configuration with a positron beam.

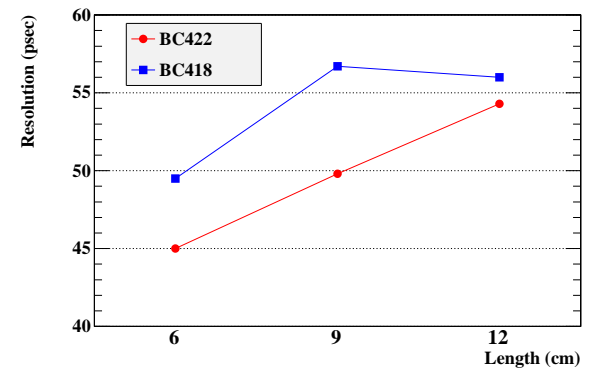

Figure 5: Scintillator comparison with several length. $\mathrm{BC} 422$ gives better resolution than $\mathrm{BC} 418$.

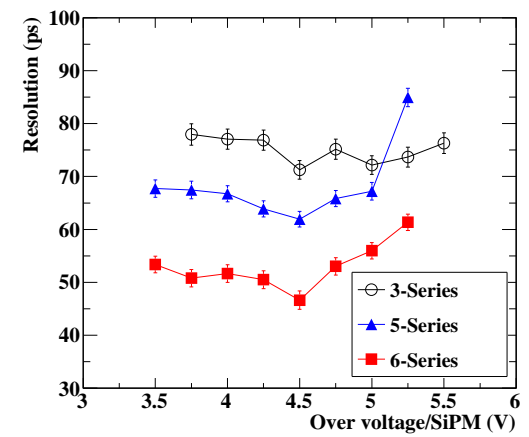

Figure 6: The number of SiPMs dependence. Counter with more SiPMs demonstrates better resolution.

\section{Summary}

We present the development of a novel pixelated timing counter for the upgraded MEG II detector. The timing counter consists of many $(\sim 500)$ small counters, each made of a scintillator counter readout by SiPMs. The resolution of the timing counter is obtained by combining multiple measurements. In the beam test, the improvement of time resolution with the number of hit counters has been demonstrated. Moreover, the configuration of the single counter has been further optimized improving its time resolution so that an improved resolution of the timing counter is expected.

\section{References}

[1] J. Adam et.al., Phys. Rev. Lett.110.201801 (2013).

[2] S. Ritt et al., NIM-A, 623, pp. 486 - 488, (2010).

[3] P.W. Cattaneo et al., IEEE Trans. Nucl. Sci., 61(5), pp. 2657 - 2666, (2014). 